\title{
Review Article \\ The Applications and Features of Liquid Chromatography-Mass Spectrometry in the Analysis of Traditional Chinese Medicine
}

\author{
Bingyao Pang, ${ }^{1}$ Ying Zhu, ${ }^{1}$ Longqing Lu, ${ }^{1}$ Fangbing Gu, ${ }^{1}$ and Hailong Chen ${ }^{2}$ \\ ${ }^{1}$ Department of Infectious Diseases, The First Affiliated Hospital of Dalian Medical University, Dalian, Liaoning, China \\ ${ }^{2}$ Department of General Surgery, The First Affiliated Hospital of Dalian Medical University, Dalian, Liaoning, China \\ Correspondence should be addressed to Ying Zhu; zhuyingsh52@126.com and Hailong Chen; hailongchen2007@hotmail.com
}

Received 2 May 2016; Accepted 12 October 2016

Academic Editor: Nunziatina De Tommasi

Copyright (c) 2016 Bingyao Pang et al. This is an open access article distributed under the Creative Commons Attribution License, which permits unrestricted use, distribution, and reproduction in any medium, provided the original work is properly cited.

\begin{abstract}
With increasingly improved separation of complex samples and detection of unknown material capabilities, liquid chromatography coupled with mass spectrometry (LC-MS) has been widely used in traditional Chinese medicine (TCM) research. This article describes the principles of liquid chromatography (LC) and mass spectrometry (MS) and their advantages and disadvantages in qualitative and quantitative analysis of TCM. We retrieved research literatures about the application of LC-MS in TCM published during the past five years at home and abroad. To better guide the analysis of TCM, this review mainly focuses on the applications category of LC-MS, how often different kinds of LC-MS are used, and the qualitative and quantitative ability of various LC-MS in the study of TCM.
\end{abstract}

\section{Introduction}

Thousands of years of accumulation of experience on life and disease made by our ancestors finally translated into a modern pharmacy. Chinese herbs, with complex and various ingredients, are usually put into practice by prescription, following the rules of monarch, minister, assistant, and guide. When applied, the amount of single herbs and pharmaceutical formulations can vary, which may lead to changes in the interaction between the drugs and the active ingredient. In different drug application sites, there will be differences in ingredients. Because of the complexity of the chemical composition of TCM, different chemical compositions may also react with each other. Although the practical application has proved the effectiveness of TCM, it is still difficult to explain the specific drug active ingredients of TCM in modern science. In recent years, a compound's in vivo metabolism and mechanism of action have become a hot Chinese medicine research topic. But its characteristics, such as complex composition, mechanism of multitarget and multimode of action, and role of ingredients, gives the research of TCM a certain degree of difficulty.

LC-MS fully integrates the high separation capacity of the sample of LC for complex samples and the strong qualitative ability of MS [1]. Because of its high sensitivity and selectivity, LC-MS technique has been widely used in TCM research [2].

\section{LC}

Chromatography separates the mixture using the differences of the distribution coefficient between the two phases (mobile and stationary phase). According to the state of the mobile phase, chromatography can be divided into gas chromatography, liquid chromatography, and supercritical fluid chromatography, while, according to the geometric forms of the stationary phase, chromatography can be divided into column chromatography, paper chromatography, and thin layer chromatography. The most commonly used LC method is column chromatography which regards liquid as a mobile phase. High performance liquid chromatography (HPLC) is modified based on the classic liquid column chromatography.

The application of LC is divided into two categories. One of them is qualitative or quantitative for a particular composition. Qualitation is managed according to the consistency between the sample and the target component in the peak time [38]. Quantitation is performed according to the 
TABLE 1: LC-Q-MS research literatures in the application of traditional Chinese medicine published during the past five years at home and abroad.

\begin{tabular}{lccc}
\hline Standards & Traditional Chinese medicine & Qualitative & Quantitative \\
\hline 5-HT and NE & Rhodioloside & + & + \\
Calycosin-7-O- $\beta$-D-glucoside, and so forth & Tou nong san & + & - \\
Salvianolic acid D & Salvianolic acid D & + & {$[3]$} \\
\hline
\end{tabular}

standard curve generated after standards are injected at different concentration levels. The other one is a fingerprint [39] which refers to the notion that, after the fingerprint sample has been disposed of in some way, we can obtain chromatogram or spectrogram labeled chemical characteristics by using certain methods of analysis.

LC has a great advantage on the capability of separating complex samples, so it is the most effective option when applied to separate mixtures, but not suitable to obtain structural information of the material [2, 40-48]. Qualitation finished by the contrast between the peak positions of unknown compounds and the standards is not available for monitoring of unknown compounds.

\section{MS}

Mass spectrometry is widely used in the field of TCM research due to its high selectivity, high sensitivity, and capability of providing information including relative molecular mass and structural characteristics. MS completes the qualitation using molecular mass and relevant structural information and completes quantitation by the relationships of the peak and compound content which the peak represented. Atmospheric pressure ionization (API) of MS has electrospray ionization (ESI) and atmospheric pressure chemical ionization (APCI) [49]. For many types of compounds, ESI has high sensitivity. Compared with ESI, APCI is suitable for the less polar compounds and the analysis of volatile compounds. Depending on the differences among mass analyzers used, common MS concludes quadrupole mass spectrum (Q-MS), time-of-flight mass spectrum (TOF-MS), and ion trap mass spectrometry (IT-MS) [50-53].

Tandem mass spectrometry refers to two or more MS working together. The most commonly used tandem mass spectrometry is triple-quadrupole mass spectrometry (QQQ-MS). In order to use quadrupole to conduct multistage mass spectrometry, three quadrupoles are sequentially placed, which is triple quadrupole [2]. Another type of tandem mass spectrometry, such as quadrupole-time-offlight mass spectrometry (Q-TOF-MS) and quadrupole-ion trap tandem mass spectrometry (Q-IT-MS), also consists of a variety of quality analyzer series [54-58]. Ion trap time series can achieve multistage MS scans sequentially at different times, so this study categorized IT-MS as tandem mass spectrometer.

Tandem mass spectrometry can induce fragments of molecular ions generated by first-stage MS, according to which we can infer the relationship between child and parent, obtain structural information of the molecule and then suggest the structure of the compound, and conduct the qualitation analysis for known and unknown compounds more accurately.

Although MS can provide structural information of a material, it requires higher purity for the sample. In TCM research, it is generally used in combination with LC.

\section{LC-MS}

LC-MS technique, using LC as a separation system and MS as a detection system, finally achieves the spectrum. When the LC and MS work together, they can carry out multistage MS to speculate the structure of the compound, thus finishing qualitative and quantitative analysis more accurately [1].

Retrieving the papers on LC-MS in the application of TCM research literatures published during the past five years at home and abroad, we found that they could be separated into two categories, that is, LC-Q-MS and LC-MS/MS. In order to analyze the differences and the advantages and disadvantages of each method, papers were classified according to the difference of tandem mass spectrometry.

4.1. LC-Q-MS. LC-Q-MS can be used to conduct qualitative and quantitative analysis for standard components, as shown in Table 1. When conducting qualitative analysis, we can obtain structural information through scanning positive or negative ions [3]. We can also make it clear which kind of substance the chemical structure belongs to by comparing it with the standard literatures [3, 4]. The LC-Q-MS quantifies compounds based on the selection of specific ions for monitoring [5]. In the absence of standards, LC-Q-MS can conduct the qualitative analysis for the substance using strong qualitative features of mass spectrometry [3].

LC-Q-MS can analyze substances qualitatively, but some isomers or identical molecular weight substances need to be further explored, whose structures require being further identified by reference to multistage mass spectrometry. Moreover, inaccurate qualitation leads to bias of quantitation.

\section{2. $L C-M S / M S$}

4.2.1. LC-IT-MS. IT-MS captures different quality range of ion making use of different sizes of RF voltage applied to the ring electrodes. It has high sensitivity, and a single IT-MS can achieve multilevel mass spectrometry functions $[6,7]$.

IT-MS can realize time-series multistage MS scans in different chronological orders, providing ingredient MSn fragmentation; thus, it is suitable for qualitative matter, 
TABLE 2: LC-IT-MS research literatures in the application of traditional Chinese medicine published during the past five years at home and abroad.

\begin{tabular}{lccr}
\hline Standards & Traditional Chinese medicine & Qualitative & Quantitative \\
\hline Danshensu and so forth & Jitai tablets & + & - \\
Baicalin and so forth & Huangqin tang & + & - \\
- & Shu-yu capsule & + & {$[6]$} \\
Ginsenoside Rgl and so forth & Si-ni decoction analogous formulae & + & - \\
Ganoderic acid A and so forth & Ganoderma lucidum & + & - \\
Protocatechuic acid and so forth & Danmu injection & + & - \\
\hline
\end{tabular}

providing structural information for the identification of unknown composition, as shown in Table 2. In the study of TCM, LC-IT-MS is commonly used in qualitative composition [6-11].

4.2.2. $L C-Q Q Q-M S$. Triple quadrupole means that three quadrupoles are orderly placed. Each quadrupole has a separate function. Scan modes of triple quadrupole include full-scan mode, product ion scan mode, parent ion scan mode, neutral loss scan mode, selected ion scan mode, and multiple reactions monitoring scan mode $[12,13]$.

LC-QQQ-MS is mainly used for quantification [12-24], as shown in Table 3. Some studies are used for qualitation $[25,26]$. Compared with LC-Q-MS, LC-QQQ-MS can select particular ions to collide and analyze the fragments after collision. LC-QQQ-MS can detect the parent ion and daughter ion at the same time, so it is accurate, sensitive, and comprehensive. LC-QQQ-MS can be applied to a wider range. Compared with other tandem mass spectrometries, triple quadrupole has the best quantitative reproducibility. LC-QQQ-MS is the most commonly known substance quantitative method.

4.2.3. LC-Q-TOF-MS. LC-Q-TOF-MS is generally used for qualitation. It has higher detection sensitivity and mass resolution. LC-Q-TOF-MS can accurately measure mass [59].

LC-Q-TOF-MS is mainly used for material qualitative analysis [27-37], as shown in Table 4. Compared with several other tandem mass spectrometries, it has advantages in detection sensitivity, mass accuracy, and resolution. High resolution and mass accuracy give LC-Q-TOF-MS better qualitative capability for fragment ions. It is also more persuasive when distinguishing ions' structure and isomers besides parsing unknown structures in this method [37].

\section{Conclusions}

Compared with LC-MS and LC-MS/MS, the research of LCMS/MS is conducted relatively more, which has a higher accuracy of qualitative analysis for known and unknown compounds. LC-IT-MS, because of its capability of multiple levels of mass spectrometry, has a better performance when assessing an unknown composition structure. LC-Q-IT-MS has a better quality of resolution than LC-IT-MS. Compared with the other three kinds of LC-MS/MS, LC-QQQ-MS was found to be more often applied for research, with a better quantitative ability than others. Its qualitative capability is good, but not as good as LC-Q-TOF-MS yet. LC-Q-TOFMS is mainly used for qualitative analysis of matter. Some of its abilities are relatively excellent, such as the sensitivity for detection, mass accuracy, and resolution.

Qualitative methods are different among different LCMS methods, while the quantitative methods are basically the same, because all quantitative methods require standard with different concentrations. Quantitative methods are usually divided into two different types: the external standard method and the internal standard method. External standard method means that, to conduct the quantitative analysis, a standard curve is generated firstly whose horizontal axis represents concentrations and vertical axis represents the color peak spectra area. Then, according to the peak area, qualitative analysis can be done. Internal standard method means the internal standard with known concentration is added to the solutions of sample and hybrid reference substance firstly; then, a standard curve is generated whose horizontal axis represents the ratio of reference sample concentration to internal standard sample concentration and vertical axis represents the ratio of standard peak area to internal standard peak area. Lastly, the internal standard sample is injected. According to the peak area of test indicators and the internal standard, qualitative analysis can be done.

In the research of TCM, it is concluded that LC-Q-TOFMS is a better choice in qualitative analysis while LC-QQQMS proves to be better for quantitative analysis. Quantitative analysis is performed using specific standard with specific concentration. Qualitative analysis can measure the structure of some unknown ingredients and speculate their attributions according to information provided by existing research literatures and database. Standard validation is the most accurate for qualitative material. Because of the complex composition and unknown abilities of TCM, research methods relatively cannot meet the need yet. Further research and exploration are still necessary.

\section{Competing Interests}

All the authors declare that they have no competing interests. 


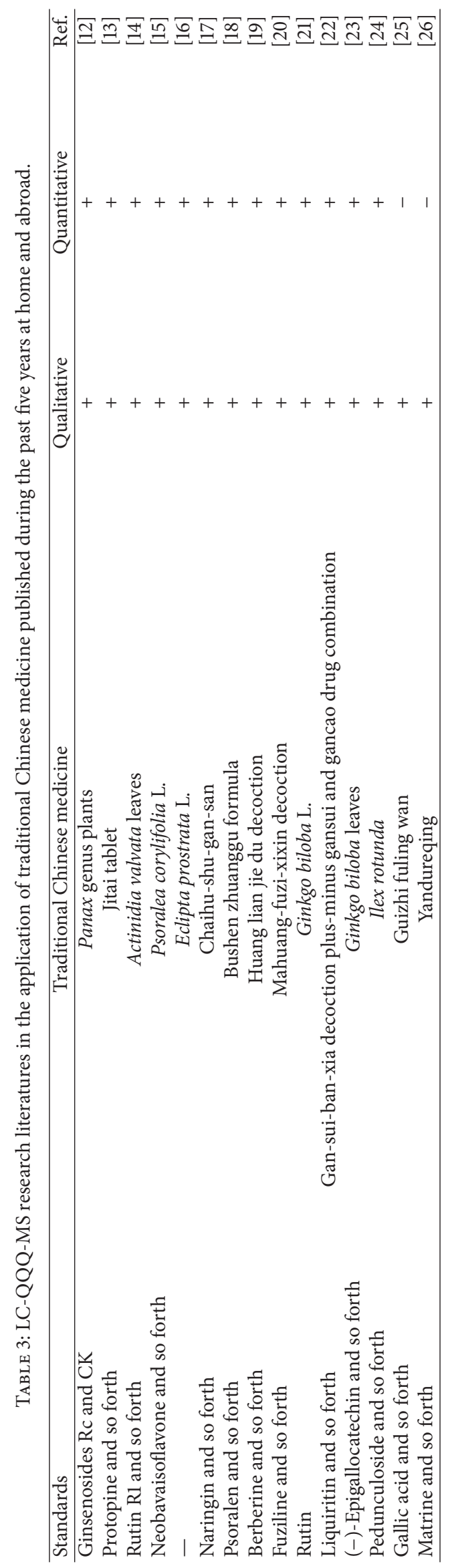


TABLE 4: LC-Q-TOF-MS research literatures in the application of traditional Chinese medicine published during the past five years at home and abroad.

\begin{tabular}{|c|c|c|c|c|}
\hline Standards & Traditional Chinese medicine & Qualitative & Quantitative & Ref. \\
\hline Fuziline and so forth & Mahuang-fuzi-xixin decoction & + & - & [20] \\
\hline Catalpol and so forth & Jieduquyuziyin prescription & + & - & [27] \\
\hline Chrysoeriol-7-O-glucoside and so forth & Lonicerae macranthoides & + & - & [28] \\
\hline Amygdalin and so forth & San-ao-tang & + & - & [29] \\
\hline Nicotinate and so forth & Saikosaponins & + & - & [30] \\
\hline Betaine and so forth & Hu-gan-kang-yuan capsules & + & - & {$[31]$} \\
\hline Chlorogenic acid and so forth & Yinhuang drop pill & + & + & {$[32]$} \\
\hline- & Bufeiyishen formula & + & - & [33] \\
\hline Ferulic acid and so forth & Rhizoma Chuanxiong & + & - & [34] \\
\hline Ononin and so forth & Guge fengtong tablet & + & - & [35] \\
\hline Columbamine and so forth & Roots of Coptis chinensis Franch. & + & - & [36] \\
\hline Grosvenorine I and so forth & Siraitiae fructus & + & + & [37] \\
\hline
\end{tabular}

\section{Acknowledgments}

This work is supported by the National Natural Science Foundation of China (no. 81273925).

\section{References}

[1] H. Wu, J. Guo, S. Chen et al., "Recent developments in qualitative and quantitative analysis of phytochemical constituents and their metabolites using liquid chromatography-mass spectrometry," Journal of Pharmaceutical and Biomedical Analysis, vol. 72, pp. 267-291, 2013.

[2] D. Steinmann and M. Ganzera, "Recent advances on HPLC/MS in medicinal plant analysis," Journal of Pharmaceutical and Biomedical Analysis, vol. 55, no. 4, pp. 744-757, 2011.

[3] X. Zhang, Q. Du, C. Liu et al., "Rhodioloside ameliorates depressive behavior via up-regulation of monoaminergic system activity and anti-inflammatory effect in olfactory bulbectomized rats," International Immunopharmacology, vol. 36, pp. 300-304, 2016

[4] L.-H. Fang, R.-P. Wang, S.-Y. Hu, Y.-H. Teng, and W.-B. Xie, "The effect of Tou Nong San on transplanted tumor growth in nude mice," Evidence-Based Complementary and Alternative Medicine, vol. 2015, Article ID 518454, 15 pages, 2015.

[5] J. Song, W. Zhang, J. Sun et al., "Pharmacokinetic study of salvianolic acid D after oral and intravenous administration in rats," Acta Pharmaceutica Sinica B, vol. 5, no. 3, pp. 246-253, 2015.

[6] S. Wang, L. Liu, L. Wang, Y. Hu, W. Zhang, and R. Liu, "Structural characterization and identification of major constituents in jitai tablets by high-performance liquid chromatography/ diode-array detection coupled with electrospray ionization tandem mass spectrometry," Molecules, vol. 17, no. 9, pp. 1047010493, 2012.

[7] T. Li, S. Zhuang, Y. Wang et al., "Flavonoid profiling of a traditional Chinese medicine formula of Huangqin Tang using high performance liquid chromatography," Acta Pharmaceutica Sinica B, vol. 6, no. 2, pp. 148-157, 2016.

[8] F. Li, Y. Zhang, X. Wei, C. Song, M. Qiao, and H. Zhang, "Metabolic profiling of Shu-Yu capsule in rat serum based on metabolic fingerprinting analysis using HPLC-ESI-MSn," Molecular Medicine Reports, vol. 13, no. 5, pp. 4191-4204, 2016.
[9] Q. Chen, S. Xiao, Z. Li, N. Ai, and X. Fan, "Chemical and metabolic profiling of $\mathrm{Si}-\mathrm{Ni}$ decoction analogous formulae by high performance liquid chromatography-mass spectrometry," Scientific Reports, vol. 5, article 11638, 2015.

[10] M. Yang, X. Wang, S. Guan et al., "Analysis of triterpenoids in ganoderma lucidum using liquid chromatography coupled with electrospray ionization mass spectrometry," Journal of the American Society for Mass Spectrometry, vol. 18, no. 5, pp. 927939, 2007.

[11] F. X. Zhu, J. J. Wang, X. F. Li, E. Sun, and X. B. Jia, “Qualitative and quantitative analysis of the major constituents in traditional Chinese medicine Danmu injection using LC-ESI-MS(n) and LC-DAD," Pharmacognosy Magazine, vol. 10, no. 39, pp. 254264, 2014.

[12] Z. Ning, Z. Liu, Z. Song et al., "A single marker choice strategy in simultaneous characterization and quantification of multiple components by rapid resolution liquid chromatography coupled with triple quadrupole tandem mass spectrometry (RRLCQqQ-MS)," Journal of Pharmaceutical and Biomedical Analysis, vol. 124, pp. 174-188, 2016.

[13] S. Wang, P. Fu, L. Liu et al., "Simultaneous determination of fifteen constituents of Jitai tablet using ultra high-performance liquid chromatography coupled with triple quadrupole electrospray tandem mass spectrometry," Molecules, vol. 19, no. 2, pp. 1635-1650, 2014

[14] Q.-H. Du, Q.-Y. Zhang, T. Han, Y.-P. Jiang, C. Peng, and H.L. Xin, "Dynamic changes of flavonoids in Actinidia valvata leaves at different growing stages measured by HPLC-MS/MS," Chinese Journal of Natural Medicines, vol. 14, no. 1, pp. 66-72, 2016.

[15] Q. Gao, Z. Xu, G. Zhao et al., "Simultaneous quantification of 5 main components of Psoralea corylifolia L. in rats' plasma by utilizing ultra high pressure liquid chromatography tandem mass spectrometry," Journal of Chromatography B: Analytical Technologies in the Biomedical and Life Sciences, vol. 1011, pp. 128135, 2016.

[16] L. Han, E. Liu, A. Kojo et al., "Qualitative and quantitative analysis of Eclipta prostrata L. by LC/MS," Scientific World Journal, vol. 2015, Article ID 980890, 15 pages, 2015.

[17] S. Q. Li, S. Dong, Z. H. Su et al., "Comparative pharmacokinetics of naringin in rat after oral administration of chaihu-shu-gansan aqueous extract and naringin alone," Metabolites, vol. 3, no. 4, pp. 867-880, 2013. 
[18] L. Pei, Y. Bao, S. Liu, J. Zheng, and X. Chen, "Material basis of Chinese herbal formulas explored by combining pharmacokinetics with network pharmacology," PLoS ONE, vol. 8, no. 2, Article ID e57414, 2013.

[19] W. Ren, R. Zuo, Y.-N. Wang et al., "Pharmacokinetic-pharmacodynamic analysis on inflammation rat model after oral administration of Huang Lian Jie Du decoction," PLOS ONE, vol. 11, no. 6, Article ID e0156256, 2016.

[20] Q. Sun, H. Cao, Y. Zhou et al., "Qualitative and quantitative analysis of the chemical constituents in Mahuang-Fuzi-Xixin decoction based on high performance liquid chromatography combined with time-of-flight mass spectrometry and triple quadrupole mass spectrometers," Biomedical Chromatography, vol. 30, no. 11, pp. 1820-1834, 2016.

[21] X. Yao, G. Zhou, Y. Tang et al., "A UPLC-MS/MS method for qualification of quercetin-3-O- $\beta$-D- glucopyranoside- $(4 \leftarrow 1)$ $\alpha$-L-rhamnoside in rat plasma and application to pharmacokinetic studies," Molecules, vol. 18, no. 3, pp. 3050-3059, 2013.

[22] Y. Zhang, D. Qian, Y. Pan et al., "Comparisons of the pharmacokinetic profile of four bioactive components after oral administration of gan-sui-ban-xia decoction plus-minus gansui and gancao drug combination in normal rats," Molecules, vol. 20, no. 5, pp. 9295-9308, 2015.

[23] Y. Zhang, J. Yuan, Y. Wang, Y. Wang, R. An, and X. Wang, "LCMS/MS determination and pharmacokinetics study of puerarin and daidzein in rat plasma after oral administration of Gegenqinlian decoction and Radix Puerariae extract," Pharmacognosy Magazine, vol. 10, no. 39, pp. 241-248, 2014.

[24] W. Zhao, L. Pang, D. Xu, and N. Zhang, "LC-MS/MS determination and pharmacokinetic study of pedunculoside in rat plasma after oral administration of pedunculoside and Ilex rotunda extract," Molecules, vol. 20, no. 5, pp. 9084-9098, 2015.

[25] L. Han, X. Guo, H. Bian et al., "Guizhi fuling wan, a traditional chinese herbal formula, sensitizes cisplatin-resistant human ovarian cancer cells through inactivation of the PI3K/AKT/ mTOR pathway," Evidence-Based Complementary and Alternative Medicine, vol. 2016, Article ID 4651949, 11 pages, 2016.

[26] Y.-J. Yang, J.-Y. Li, X.-W. Liu, J.-Y. Zhang, Y.-R. Liu, and B. Li, “A non-biological method for screening active components against influenza virus from traditional Chinese medicine by coupling a LC column with oseltamivir molecularly imprinted polymers," PLoS ONE, vol. 8, no. 12, Article ID 0084458, 2013.

[27] X. Ding, J. Hu, C. Wen, Z. Ding, L. Yao, and Y. Fan, "Rapid resolution liquid chromatography coupled with quadrupole timeof-flight mass spectrometry-based metabolomics approach to study the effects of jieduquyuziyin prescription on systemic lupus erythematosus," PLoS ONE, vol. 9, no. 2, article e88223, 2014.

[28] X. Hu, L. Chen, S. Shi, P. Cai, X. Liang, and S. Zhang, "Antioxidant capacity and phenolic compounds of Lonicerae macranthoides by HPLC-DAD-QTOF-MS/MS," Journal of Pharmaceutical and Biomedical Analysis, vol. 124, pp. 254-260, 2016.

[29] C. Ma, Y. Qian, X. Fan, E. Shang, X. Yao, and S. Ma, "Using UPLC-QTOF-MS to analyze the chemical changes between traditional and dispensing granule decoctions of san-ao," Journal of Chromatographic Science, vol. 52, no. 4, pp. 277-292, 2014.

[30] Y. Ma, Y. Bao, S. Wang et al., "Anti-inflammation effects and potential mechanism of saikosaponins by regulating nicotinate and nicotinamide metabolism and arachidonic acid metabolism," Inflammation, vol. 39, no. 4, pp. 1453-1461, 2016.
[31] F. Wei, M. Chen, C. Luo, F. Chen, Q. Shen, and Z. Mo, “Developing an absorption-based quality control method for Hu-GanKang-Yuan capsules by UFLC-QTOF-MS/MS screening and HPLC-DAD quantitative determination," Molecules, vol. 21, no. 5, article 592, 2016.

[32] T. L. Wong, Y. Q. An, B. C. Yan et al., "Comprehensive quantitative analysis of Chinese patent drug YinHuang drop pill by ultra high-performance liquid chromatography quadrupole time of flight mass spectrometry," Journal of Pharmaceutical and Biomedical Analysis, vol. 125, pp. 415-426, 2016.

[33] L. Yang, J. Li, Y. Li et al., "Identification of metabolites and metabolic pathways related to treatment with Bufei Yishen formula in a rat COPD model using HPLC Q-TOF/MS," Evidence-Based Complementary and Alternative Medicine, vol. 2015, Article ID 956750, 9 pages, 2015.

[34] T. Yi, J. Y. Fang, L. Zhu et al., "The variation in the major constituents of the dried rhizome of Ligusticum chuanxiong (Chuanxiong) after herbal processing," Chinese Medicine, vol.11, article 26, 2016.

[35] S.-L. Zeng, P. Li, and E.-H. Liu, "Metabolic profile of Guge Fengtong tablet in rat urine and bile after oral administration, using high-performance liquid chromatography coupled with electrospray ionization quadrupole time-of-flight mass spectrometry," Chinese Journal of Natural Medicines, vol. 13, no. 12, pp. 954-960, 2015.

[36] H. Zhao, S. Zhou, M. Zhang et al., "An in vitro AChE inhibition assay combined with UF-HPLC-ESI-Q-TOF/MS approach for screening and characterizing of AChE inhibitors from roots of Coptis chinensis Franch," Journal of Pharmaceutical and Biomedical Analysis, vol. 120, pp. 235-240, 2016.

[37] G. Zhou, M. Wang, Y. Li, R. Xu, and X. Li, "Comprehensive analysis of 61 characteristic constituents from Siraitiae fructus using ultrahigh-pressure liquid chromatography with timeof-flight mass spectrometry," Journal of Pharmaceutical and Biomedical Analysis, vol. 125, pp. 1-14, 2016.

[38] D.-H. Li, Y.-S. Lv, J.-H. Liu et al., "Simultaneous determination of four active ingredients in Sargentodoxa cuneata by HPLC coupled with evaporative light scattering detection," International Journal of Analytical Chemistry, vol. 2016, Article ID 8509858, 7 pages, 2016.

[39] Z. Zhang, Y. Zhang, Z. Zhang et al., "Comparative analysis of DNA barcoding and HPLC Fingerprint to trace species of phellodendri cortex, an Important Traditional Chinese Medicine from multiple sources," Biological \& Pharmaceutical Bulletin, vol. 39, no. 8, pp. 1325-1330, 2016.

[40] L. J. Liu and J. N. Liu, "A strategy for quality control of Menispermum dauricum DC based on cytotoxic activity and HPLC fingerprint analysis," Indian Journal of Pharmaceutical Sciences, vol. 78, no. 1, pp. 143-150, 2016.

[41] B. Hazra, M. Das Sarma, and U. Sanyal, "Separation methods of quinonoid constituents of plants used in Oriental traditional medicines," Journal of Chromatography B: Analytical Technologies in the Biomedical and Life Sciences, vol. 812, no. 1-2, pp. 259$275,2004$.

[42] S. Hisiger and M. Jolicoeur, "Analysis of Catharanthus roseus alkaloids by HPLC," Phytochemistry Reviews, vol. 6, no. 2-3, pp. 207-234, 2007.

[43] W. Oleszek and Z. Bialy, "Chromatographic determination of plant saponins-an update (2002-2005)," Journal of Chromatography $A$, vol. 1112, no. 1-2, pp. 78-91, 2006. 
[44] S.-C. Wang, T.-Y. Tseng, C.-M. Huang, and T.-H. Tsai, "Gardenia herbal active constituents: applicable separation procedures," Journal of Chromatography B: Analytical Technologies in the Biomedical and Life Sciences, vol. 812, no. 1-2, pp. 193-202, 2004.

[45] O. Sticher, "Natural product isolation," Natural Product Reports, vol. 25, no. 3, pp. 517-554, 2008.

[46] H.-Y. Zhao and J.-G. Jiang, "Application of chromatography technology in the separation of active components from nature derived drugs," Mini Reviews in Medicinal Chemistry, vol. 10, no. 13, pp. 1223-1234, 2010.

[47] E. S. Ong, "Extraction methods and chemical standardization of botanicals and herbal preparations," Journal of Chromatography B: Analytical Technologies in the Biomedical and Life Sciences, vol. 812, no. 1-2, pp. 23-33, 2004.

[48] W. Li, Z. Chen, Y. Liao, and H. Liu, "Separation methods for toxic components in Traditional Chinese Medicines," Analytical Sciences, vol. 21, no. 9, pp. 1019-1029, 2005.

[49] W. A. Korfmacher, "Foundation review: principles and applications of LC-MS in new drug discovery," Drug Discovery Today, vol. 10, no. 20, pp. 1357-1367, 2005.

[50] J. W. Allwood and R. Goodacre, "An introduction to liquid chromatography-mass spectrometry instrumentation applied in plant metabolomic analyses," Phytochemical Analysis, vol. 21, no. 1, pp. 33-47, 2010.

[51] D. J. Douglas, A. J. Frank, and D. Mao, "Linear ion traps in mass spectrometry," Mass Spectrometry Reviews, vol. 24, no. 1, pp. 129, 2005.

[52] Q. Hu, R. J. Noll, H. Li, A. Makarov, M. Hardman, and R. G. Cooks, "The Orbitrap: a new mass spectrometer," Journal of Mass Spectrometry, vol. 40, no. 4, pp. 430-443, 2005.

[53] A. G. Marshall and C. L. Hendrickson, "High-resolution mass spectrometers," Annual Review of Analytical Chemistry, vol. 1, no. 1, pp. 579-599, 2008.

[54] G. L. Glish and D. J. Burinsky, "Hybrid mass spectrometers for tandem mass spectrometry," Journal of the American Society for Mass Spectrometry, vol. 19, no. 2, pp. 161-172, 2008.

[55] X.-Y. Guo, J. Han, M. Ye et al., "Identification of major compounds in rat bile after oral administration of total triterpenoids of Ganoderma lucidum by high-performance liquid chromatography with electrospray ionization tandem mass spectrometry," Journal of Pharmaceutical and Biomedical Analysis, vol. 63, pp. 29-39, 2012.

[56] S. Jarussophon, S. Acoca, J.-M. Gao et al., "Automated molecular formula determination by tandem mass spectrometry (MS/ MS)," The Analyst, vol. 134, no. 4, pp. 690-700, 2009.

[57] Y. Konishi, T. Kiyota, C. Draghici et al., "Molecular formula analysis by an MS/MS/MS technique to expedite dereplication of natural products," Analytical Chemistry, vol. 79, no. 3, pp. 1187-1197, 2007.

[58] M. Shibano, A.-S. Lin, H. Itokawa, and K.-H. Lee, "Separation and characterization of active flavonolignans of Silybum marianum by liquid chromatography connected with hybrid iontrap and time-of-flight mass spectrometry (LC-MS/IT-TOF)," Journal of Natural Products, vol. 70, no. 9, pp. 1424-1428, 2007.

[59] T. Kind and O. Fiehn, "Advances in structure elucidation of small molecules using mass spectrometry," Bioanalytical Reviews, vol. 2, no. 1, pp. 23-60, 2010. 


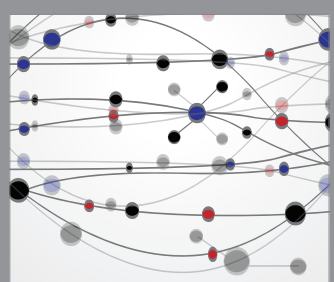

The Scientific World Journal
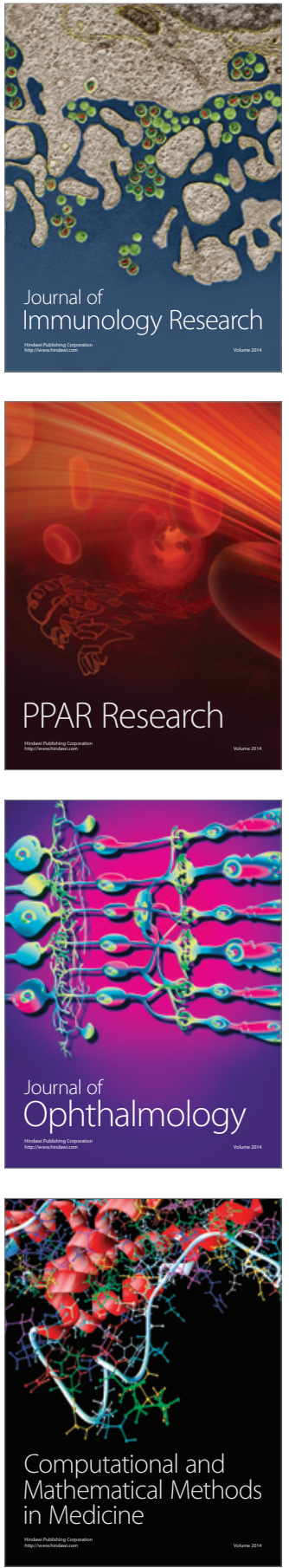

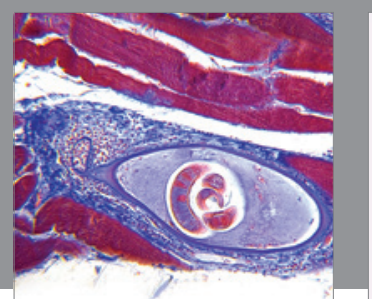

Gastroenterology Research and Practice

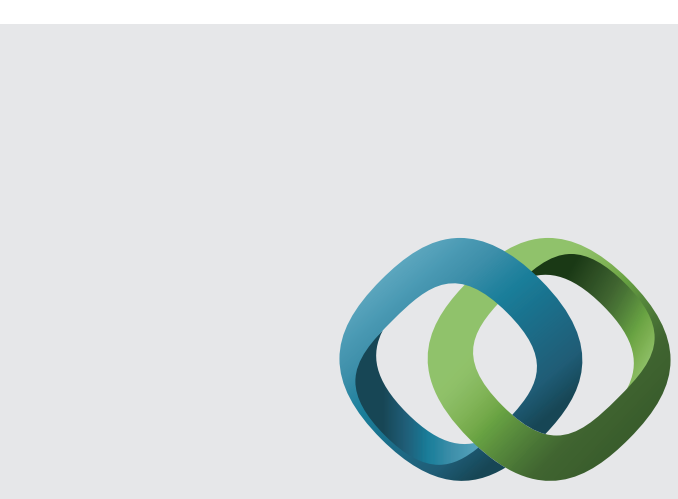

\section{Hindawi}

Submit your manuscripts at

http://www.hindawi.com
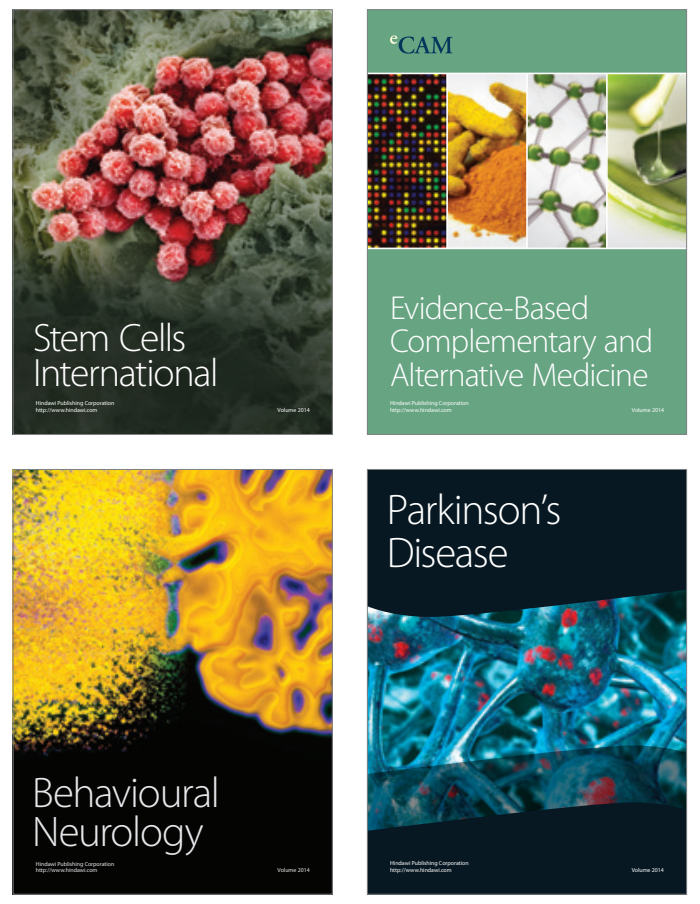
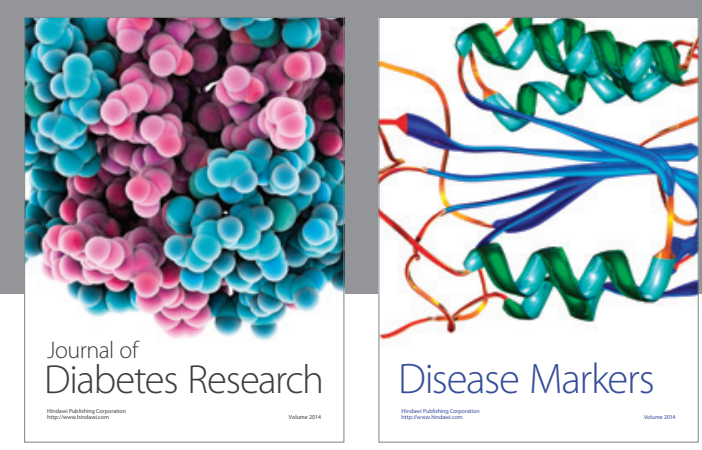

Disease Markers
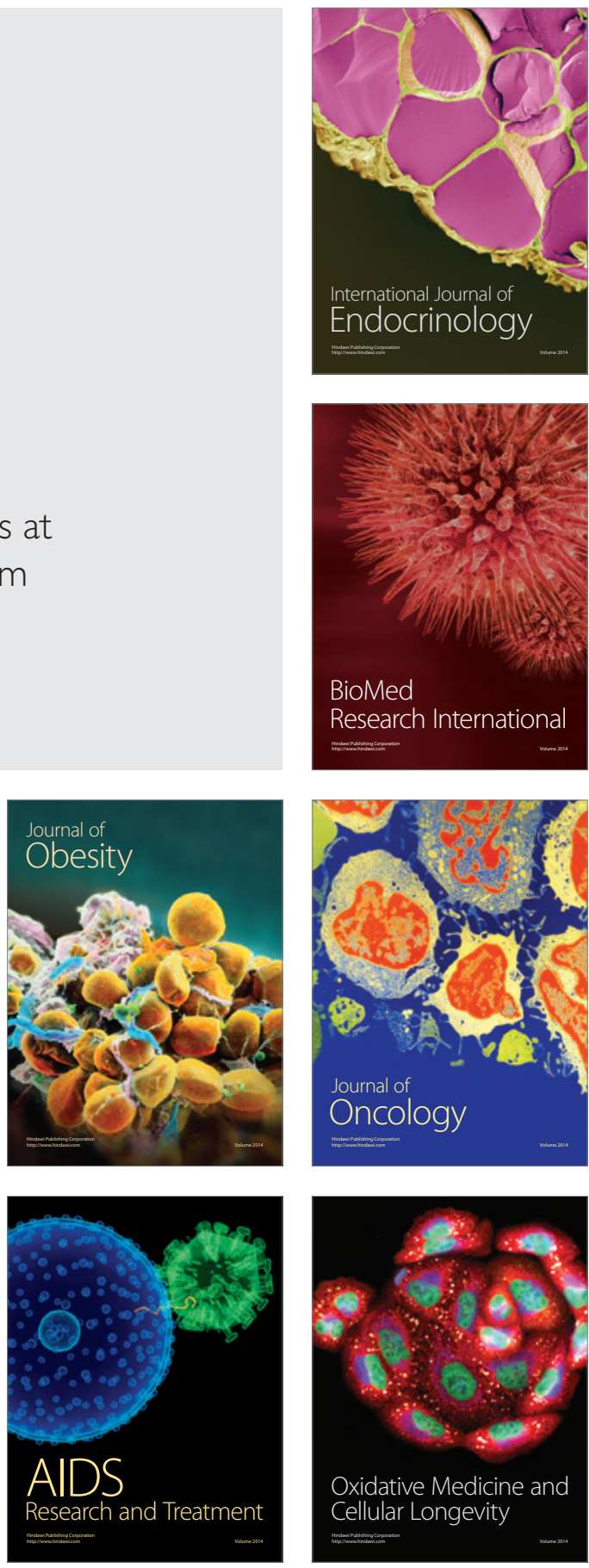